\title{
Identity Verification from Photographs in Travel Documents: The Role of Display Duration and Orientation on Performance
}

\author{
Sarah D. Chiller-Glaus ${ }^{1}$ \\ Adrian Schwaninger ${ }^{1,2}$ \\ Franziska Hofer ${ }^{1}$ \\ ${ }^{1}$ University of Zurich, Switzerland \\ ${ }^{2}$ Max Planck Institute for Biological Cybernetics, Tübingen, Germany
}

\begin{abstract}
At border control, it is the personnel's job to identify possible passport fraud, in particular to verify whether the photograph in a travel document matches its bearer. However, as various earlier studies suggest, identity verification from photographs or CCTV is far from accurate. The aim of this study was thus to investigate identity verification at border control. Particularly, we examined the influence of display duration in document verification. Results showed that performance significantly suffered from time restrictions, which stresses the importance of working environments at border control free of time pressure. A second aim was to assess a possible benefit of inversion of the document on identity verification performance, as was suggested by anecdotal evidence from security personnel but clearly contradicts the well known inversion effect in face recognition. Indeed, no such beneficial influence of inversion was found in this study. The results are discussed in terms of application-oriented implications.
\end{abstract}

\section{INTRODUCTION}

At border control and customs, the security personnel's task is to assess if the photograph in a travel document matches its bearer, and to identify any possible document fraud, e.g., when the bearer travels with the document of a similarly looking double. Findings of previous research, however, revealed that identity verification from pictures is far from flawless. A striking demonstration of this issue was made by Kemp, Towell, and Pike (1997). In their field experiment, they informed cashiers in a supermarket that a number of shoppers would present them with fraud credit cards depicting another person than the bearer. Despite increased attention to detect the impostors, the cashiers still accepted slightly over $50 \%$ of the fraud credit cards as valid. Also, it is well known that eyewitnesses to crimes often make mistakes at the attempt to identify a face: Bruce, Henderson, Greenwood, Hancock, Burton, and Miller (1999) showed that the verification of identity from unfamiliar faces captured on video leads to a high percentage of false responses. Another study revealed that even skilled personnel such as police officers experienced in forensic identification did not perform better than inexperienced persons when identifying the photograph of a face from the still image of a surveillance camera (Burton, Wilson, Cowan, \& Bruce, 1999).

Taken together, previous research implies substantial difficulties when people have to verify the identity of a person by a photograph. This ability might further be limited by time pressure in situations of document control.

An ever increasing number of passengers in aviation requires fast and smooth processing. Individual reports from security personnel at airports revealed that they have usually only a few seconds of time to assess travel documents. This includes the verification of the document photograph plus a number of other factors of authenticity.

The main aim of this study is to measure baseline performance of identity verification and to investigate the influence of time pressure upon this task. A second aim concerns the verification of anecdotal evidence from different airports about an unorthodox practice at border control: As a number of security inspectors revealed, the inversion of the document photograph together with an up-to-date picture of the passenger is supposed to facilitate identification - a procedure which utterly contradicts theoretical findings in face recognition: Yin (1969) was the first to describe that upside-down faces are disproportionately more difficult to recognize than other inverted objects, a finding which is generally referred to as face inversion effect. Subsequent replications of Yin's study have confirmed this result (e.g., Farah, Tanaka, \& Drain, 1995; Goldstein \& Chance, 1981; see Valentine, 1988, for a review). The possible benefit of inversion on identity verification performance shall thus be investigated in this study as well. 


\section{METHODS}

\section{Participants}

Seventy-two undergraduate students (43 female) from the University of Zurich participated in the experiment in exchange for course credit. The average age was 24.23 years. All had normal or corrected to normal vision and were naïve as to the purpose of this study.

\section{Materials}

As stimuli, we used up-to-date color photographs of 20 pairs of siblings (ten female) aged 16 to 55, depicting the face in frontal view with neutral expression. In addition, we used their valid document photograph (either passport or identity card, validity up to ten years). Using document photographs provided a challenging test, since a face is likely to change during the validity period of a document. The variance in appearance and age of the stimuli revealed obvious potential for difficulty in document verification. Trials were created as follows: From each pair of siblings, consisting each of person $\mathrm{A}$ and person $\mathrm{B}$, four combinations were generated so that both the up-to-date photograph of person $\mathrm{A}$ and $\mathrm{B}$ were displayed once next to their own document photograph, and once next to their sibling's, on a black background, covering approximately $12^{\circ}$ of the visual angle in height (see Figure 1).

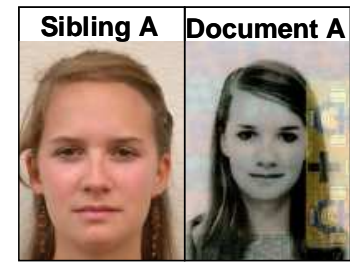

a)

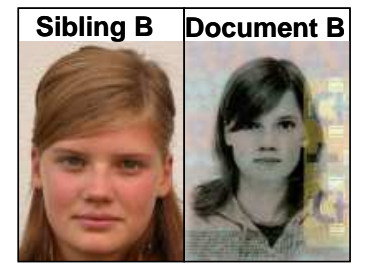

c)

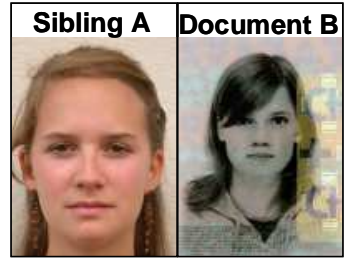

b)

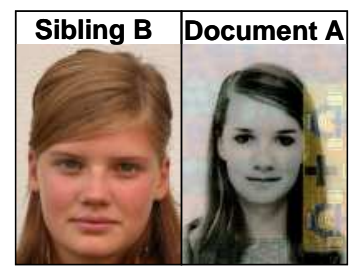

d)
Figure 1 Example of stimuli: Four combinations of up-to-date photograph and document photograph. a) and c) represent "same"trials, b) and d) "different"-trials.

\section{Procedure}

The participants' task was to decide whether the two pictures presented depicted the same person or not (simultaneous same/different matching task). Instructions on the procedure were given in written form on the monitor. Response was given by a mouse pressing one of two buttons on the screen labeled "same" or "different". To examine effects of time pressure at border control, the display duration of the stimuli was varied as follows: one second, four seconds, and self-paced. Participants were randomly assigned to one of these three conditions (24 participants to each group). All trials were presented twice, once upright and once inverted, resulting in a total of 160 trials ( 20 pairs $* 4$ combinations $* 2$ orientations). All varying factors (orientation, combination of persons A and B, gender of siblings, document type) were counterbalanced across participants using a latin square design. The independent variables were display duration (one second, four seconds, self-paced) and orientation (upright, inverted). The dependent variables were the hit and false alarm rate (a hit was defined as the correct identification of a "same"-trial, a miss as erroneous assumption that a "different"-trial depicted the same person). Performance was measured by calculating A' (for details on A', see Macmillan \& Creelman, 1991). In addition, the participants' rating of confidence about their response on a slider from "unsure" to "sure" on a 90 point scale were analyzed. Participants did not know the ratio of same and different trials, and did not receive any feedback on the correctness of their responses.

\section{RESULTS}

Results on the mean detection performance A' of upright and inverted photographs under different display durations are shown in Figure 2. Overall detection performance was relatively low, which confirms the findings of previous studies on face recognition from photographs (see introduction). A two-way ANOVA with the within-participant factor orientation (upright and inverted) and the between-participant factor display duration (one second, four seconds, and self-paced) revealed a main effect of both factors (orientation: $F(1$, $69)=98.73, p<0.001, \eta^{2}=0.59$; display duration: $F(2$, 69) $\left.=17.34, p<0.001, \eta^{2}=0.34\right)$. No interaction was found between the two factors $(F(2,69)=0.25, p=.78$, $\left.\eta^{2}=0.01\right)$, which indicates that the relative impact of orientation did not differ between the three display duration conditions. 


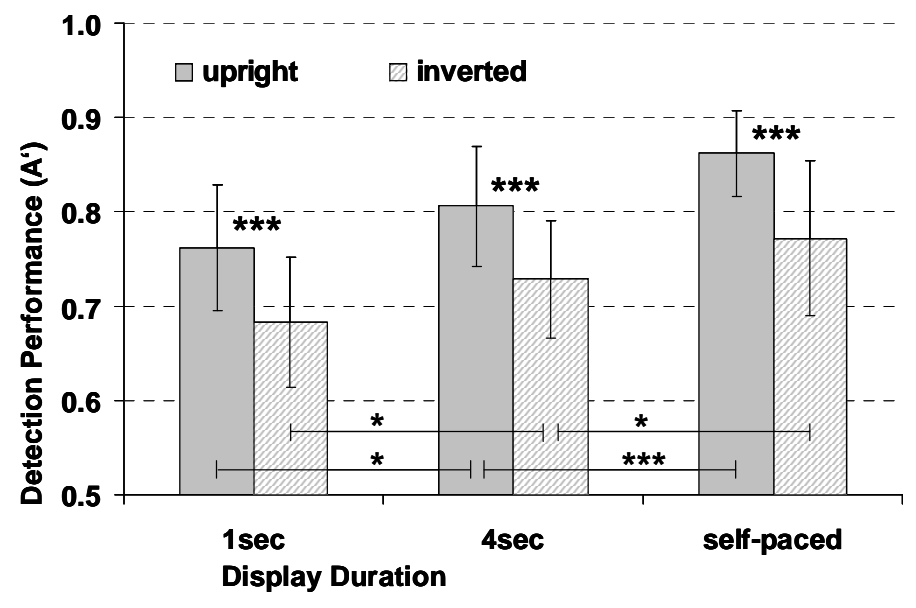

Figure 2 Influence of orientation and display duration on mean verification performance. Error bars represent standard deviations. * $=$ $p<.05, * *=p<.01, * * *=p<.001$

Paired sample t-tests (two-tailed) pointed out that the higher A' scores for upright faces were mainly the result of a lower false alarm rate, i.e. less erroneous "same" responses in the upright than in the inverted condition, while the hit rate - correct "same" responses - did not change considerably between the two orientations (see Table 1).

\begin{tabular}{l|lll} 
& $1 \mathrm{sec}$ & $4 \mathrm{sec}$ & self-paced \\
\hline Hit & $2.13 *$ & 0.58 & 1.99 \\
FA & $3.59 * *$ & $4.55 * * *$ & $9.05 * * *$
\end{tabular}

Table $1 \mathrm{t}$-values for the difference in detection performance (Hit and False Alarm Rate $=$ FA) between upright and inverted stimuli. * $=$ $p<.05, * *=p<.01, * * *=p<.001$

Identity verification performance significantly rose with increasing display duration, which stresses the importance of a working environment free of time pressure at border control. Also, in all three display duration conditions, performance was better for upright than inverted stimuli, mirroring a classical face inversion effect. This raises the question why then, in some cases, inversion of the document was reported to facilitate identity verification. To test the possibility that these reports were only the result of increased subjective confidence rather than objectively measurable performance enhancement, a two-factor ANOVA (duration and orientation) of the participants' confidence ratings was conducted. A main effect of orientation $(F(1$, $\left.69)=8.46, p<.01, \eta^{2}=0.11\right)$ revealed that participants were more confident with their judgment about upright faces, ruling out the possibility of increased confidence in the inverted condition.

\section{DISCUSSION}

The results confirm previous evidence that identity verification from photographs is to a high degree errorprone. Even without time pressure, A' for upright faces did not exceed .86. Bearing in mind that the recognition of a face is a very common task, the relatively low performance in identity verification of faces is striking.

The results also show that time pressure has a negative effect on identity verification performance: A' drops significantly when collected under a restricted display duration of four seconds, and is reduced even further in the condition of one second display duration. This could be critical since usually only a few seconds are used for the verification of the whole travel document including the picture of the person. We are currently investigating whether experienced security personnel perform better than the student participants tested in this study.

Regarding the inversion of the photographs, our results show that in average, turning the document upside down does not improve, but in fact reduce performance. This is contrary to the claims of individual security officers according to which identification is easier from inverted documents. In our sample, a subjective feeling of increased confidence with inverted faces as a possible explanation for this practice could be ruled out by analyzing the confidence ratings. On an individual level, however, we found that roughly $7 \%$ of the participants performed contrary to the majority and showed better results for inverted photographs. Additional research is needed to find out whether there really is a small percentage of people who can perform face picture matching consistently better when faces are inverted.

Furthermore, performance on the individual level revealed large differences among participants, especially in the conditions where display duration was limited. Such large standard deviations among participants might imply individually developed matching abilities or the use of particular strategies. If such can be found consistently, it would be beneficial to develop preemployment assessment tests to select candidates who are well-suited for the document verification task.

Identity verification is a highly relevant task at thousands of airports world-wide. The results found in this study suggest that identity verification using picture ID documents might be more difficult than many people expected. In addition, there are additional aspects that should be examined, e.g. the ability of security personnel to process other-race faces, which is considered to be particularly difficult, as earlier face recognition studies suggest (see e.g., Meissner \& Brigham, 2001). We are currently conducting a series of experiments to address 
these questions in order to fill the gap of research regarding identity verification in travel documents.

\section{REFERENCES}

Bruce, V., Henderson, Z., Greenwood, K., Hancock, P.J.B., Burton, A.M., \& Miller, P. (1999). Verification of face identities from images captured on video. Journal of Experimental Psychology: Applied, 5, 4, 339-360.

Burton, A.M., Wilson, S., Cowan, M., \& Bruce, V. (1999). Face recognition in poor quality video: evidence from security surveillance. Psychological Science, 10, 243-248.

Farah, M.J., Tanaka, J.W., \& Drain, H.M., (1995). What causes the face inversion effect. Journal of Experimental Psychology: Human Perception and Performance, 21, 3, 628-634.

Goldstein, A.G., \& Chance, J.E. (1980). Memory for faces and schema theory. Journal of Psychology, 105, 47- 59.

Kemp, R., Towell, N., \& Pike, G. (1997). When seeing should not be believing: photographs, credit cards and fraud. Applied Cognitive Psychology, 11, 211-222.

Macmillan, N.A., \& Creelman, C.D. (1991). Detection Theory: A user's guide. Cambridge: Cambridge University Press.

Meissner, C.A., \& Brigham, J.C. (2001). Thirty Years of investigating the own-race bias in memory for faces: A meta-analytic review. Psychology, Public Policy, and Law, 7, 1, 3-35.

Valentine, T. (1988). Upside-down faces: A review of the effect of inversion upon face recognition. British Journal of Psychology, 79, 471-491.

Yin, R.K. (1969). Looking at upside down faces. Journal of Experimental Psychology, 81, 1, 141-145. 